\title{
Realization of N-Type Semiconducting of Phosphorene through Surface Metal Doping and Work Function Study
}

\author{
Haocheng Sun, ${ }^{1,2}$ Yuan Shang, ${ }^{1}$ Yanmei Yang $\mathbb{D}^{3},{ }^{3}$ and Meng Guo $\mathbb{D}^{1}$ \\ ${ }^{1}$ Shandong Computer Science Center (National Supercomputer Center in Jinan) and Shandong Provincial Key Laboratory of Computer \\ Networks, Qilu University of Technology (Shandong Academy of Sciences), Jinan, Shandong 250101, China \\ ${ }^{2}$ Shengli No. 1 Middle School, Dongying, Shandong 257027, China \\ ${ }^{3}$ College of Chemistry, Chemical Engineering and Materials Science, Collaborative Innovation Center of Functionalized Probes for \\ Chemical Imaging in Universities of Shandong, Key Laboratory of Molecular and Nano Probes, Ministry of Education, Shandong \\ Provincial Key Laboratory of Clean Production of Fine Chemicals, Shandong Normal University, Shandong 250014, China
}

Correspondence should be addressed to Yanmei Yang; yym@sdnu.edu.cn and Meng Guo; guomeng@sdas.org

Received 16 October 2017; Accepted 11 December 2017; Published 30 January 2018

Academic Editor: Giuseppe Compagnini

Copyright (C) 2018 Haocheng Sun et al. This is an open access article distributed under the Creative Commons Attribution License, which permits unrestricted use, distribution, and reproduction in any medium, provided the original work is properly cited.

\begin{abstract}
Phosphorene becomes an important member of the layered nanomaterials since its discovery for the fabrication of nanodevices. In the experiments, pristine phosphorene shows $p$-type semiconducting with no exception. To reach its full capability, $n$-type semiconducting is a necessity. Here, we report the electronic structure engineering of phosphorene by surface metal atom doping. Five metal elements, $\mathrm{Cu}, \mathrm{Ag}, \mathrm{Au}, \mathrm{Li}$, and $\mathrm{Na}$, have been considered which could form stable adsorption on phosphorene. These elements show patterns in their electron configuration with one valence electron in their outermost $s$-orbital. Among three group 11 elements, $\mathrm{Cu}$ can induce $n$-type degenerate semiconducting, while $\mathrm{Ag}$ and $\mathrm{Au}$ can only introduce localized impurity states. The distinct ability of $\mathrm{Cu}$, compared to $\mathrm{Ag}$ and $\mathrm{Au}$, is mainly attributed to the electronegativity. Cu has smaller electronegativity and thus denotes its electron to phosphorene, upshifting the Fermi level towards conduction band, resulting in $n$-type semiconducting. $\mathrm{Ag}$ and Au have larger electronegativity and hardly transfer electrons to phosphorene. Parallel studies of Li and Na doping support these findings. In addition, Cu doping effectively regulates the work function of phosphorene, which gradually decreases upon increasing $\mathrm{Cu}$ concentration. It is also interesting that Au can hardly change the work function of phosphorene.
\end{abstract}

\section{Introduction}

Since 2004 when graphene, the prototype two dimensional (2D) material, was realized in the experiment [1,2], enormous attention has been paid to the preparation, characterization, and application studies of 2D family members [3-10]. These atomic-thin layers are believed to find applications in the fabrications of heterojunction and logic devices with high performance working at nanoscale [3]. Silicene [11], hexagonal boron nitride [12], transition metal dichalcogenide $[9,10$, 13], and so forth have been well studied because of their novel mechanical, electronic, and photonic properties induced by the reduced dimension and thickness. With promising applications in nanoelectronics and optoelectronics, 2D material becomes to be an exciting and important research area in the recent decade.
Recently, phosphorene-the few layer and monolayer version of black phosphorus-was isolated by mechanical exfoliation from black phosphorus crystals and attracted great research interest [14-20]. Black phosphorus has been utilized in lithium-ion battery and has high capacity as well as good reversibility [21-25]. In contrast to bulk semiconductors, phosphorene has a thickness dependent bandgap $[14,15]$. In detail, the bandgap of monolayer phosphorene is $1.51 \mathrm{eV}$, while five-layer phosphorene has a bandgap of only $0.59 \mathrm{eV}$. The bandgap further decreases with increase of the thickness and reaches a value of $0.33 \mathrm{eV}$ for the bulk $[14,17]$. The bandgap of few-layer phosphorene is in a moderate range, making it a natural choice for circuit design in nanoelectronics, such as heterojunction and logic devices integrated with other 2D materials. Demonstrative field-effect transistors (FET) of few-layer phosphorene were reported 
with high charge carrier mobility, drain current modulation on the order of $10^{5}$, and well-developed current saturation in the $I-V$ measurements $[16,17]$. Generally, the pristine phosphorene always displays $p$-type semiconducting from the aforementioned FET experiments [16, 17], phosphorene$\mathrm{MoS}_{2}$ heterojunction $p-n$ diode experiment [26], and theoretical prediction [14] with no exception. In order to achieve the full capabilities of phosphorene in the logic design (like $p-n$ diode and CMOS), it is highly desirable to realize the counterpart, the $n$-type semiconducting phosphorene.

Recently, Koenig et al. reported that $\mathrm{Cu}$ doped phosphorus field-effect transistor (FET) demonstrated an $n$-type semiconducting character [27]. While doping is the most mature way in the fabrications of silicon devices, it is still unknown whether other metal elements, especially the other members of group 11, can also tune the conductivity of phosphorene. For instance, Au has been well documented to be robust to modulate the electrical and optical properties of $2 \mathrm{D}$ nanostructures [28-32], but less is known about phosphorene with Au doping. The mechanical study of foreign elemental doping to phosphorene can greatly benefit and guide the future study in this area.

Devoting our efforts, we have systematically explored the regulation of phosphorene electronic structures through doping of phosphorene by adsorption of metal atom of $\mathrm{Cu}$, $\mathrm{Ag}, \mathrm{Au}, \mathrm{Li}$, and $\mathrm{Na}$. The common feature of these five elements is that they have one valence electron occupying the outmost $s$-orbital. Three group 11 elements $(\mathrm{Cu}, \mathrm{Ag}$, and $\mathrm{Au})$ are widely used for wire-bonding and electrode materials because of low electrical resistivity. It is interesting that phosphorene doped with $\mathrm{Cu}$ displays $n$-type degenerate semiconducting. For Ag and $\mathrm{Au}$, they only lead to the formation of localized impurity states in the bandgap. We attribute the distinct ability of $\mathrm{Cu}$, compared to $\mathrm{Ag}$ and $\mathrm{Au}$, to its lower electronegativity. $\mathrm{Cu}$ has the smallest electronegativity and can easily denote its $4 \mathrm{~s}$-electron to phosphorene, effectively shifting the $E_{F}$ to the bottom of conduction band region and resulting in the $n$ type semiconducting. On the contrary, Ag and Au have larger electronegativity (Au's electronegativity is even larger than P) and can hardly transfer electrons to phosphorene. The verification studies of the two alkali elements, $\mathrm{Li}$ and $\mathrm{Na}$, also demonstrate $n$-type semiconducting because $\mathrm{Li}$ and $\mathrm{Na}$ are prone to transfer their valence electron to phosphorene; this is consistent with $\mathrm{Cu}$ doping and supports our hypothesis. Further work function studies have demonstrated that $\mathrm{Cu}$ doping can effectively regulate the work function of phosphorene, while $\mathrm{Au}$ is almost incapable of changing phosphorene's work function. Our present results open new possibilities for the preparation of phosphorene materials with diverse electronic properties and surely deserve further experimental efforts in the future.

\section{Computational Details}

The calculations were performed using Vienna ab initio simulation package (VASP) [33, 34]. Projector-augmentedwave (PAW) potentials [35] were used to take into account the electron-ion interactions, while the electron exchangecorrelation interactions were treated using generalized gradient approximation (GGA) [36] in the scheme of PerdewBurke-Ernzerhof. A plane wave cutoff of $500 \mathrm{eV}$ was used for all the calculations. All atomic positions and lattice vectors were fully optimized using a conjugate gradient algorithm to obtain the unstrained configuration. Atomic relaxation was performed until the change of total energy was less than $0.01 \mathrm{meV}$ and all the forces on each atom were smaller than $0.01 \mathrm{eV} / \AA$. Generally, the model contains one metal atom on $4 \times 3$ primitive cells of phosphorene; thus the atomic concentration of adsorbed metal is $2.08 \%$. For Cu doping, we have further considered two higher concentrations: one $\mathrm{Cu}$ in $3 \times 2$ supercell and $2 \times 1$ supercell. These correspond to $\mathrm{Cu}$ concentrations of $4.16 \%$ and $12.5 \%$, respectively. A vacuum space of $20 \AA$ was placed in the normal direction to avoid mirror interactions. $K$-point sampling of $5 \times 5 \times 1$ was used for the structure relaxation, while denser mesh of $15 \times 15 \times 1$ was used to calculate electron densities and band structures.

\section{Results and Discussion}

3.1. Metal Binding Site and Energy. Being distinct from graphene with a planar structure, phosphorene monolayer has a puckered structure with $\mathrm{P}$ atoms arranged in a honeycomb lattice as shown in Figures 1(a) and 1(b). This structural anisotropy affects the metal atoms binding and migration on the surface [25]. We first explored the phosphorene surface for metal binding by placing one metal atom at different sites above phosphorene followed by full structural optimization. The most stable binding site is above the groove between two P "clifftops" as shown in Figures 1(a) and 1(b). For $\mathrm{Li}$, a previous study has explored the phosphorene surface by performing migration pathway energy profiling with the nudged elastic band method, which has predicted the similar binding site to current study [25]. We calculated the structural configurations (as labelled in Figures 1(a) and 1(b)) between metal atom and phosphorene, and the results are shown in Table 1. Structurally, $\mathrm{Cu}$ forms the most ultimate binding with phosphorene atoms, corresponding to the smallest distance to adjacent $\mathrm{P}$ atoms. On the contrary, $\mathrm{Na}$ binds to phosphorene with the largest separation. In order to quantitatively evaluate the binding stability, we calculated the binding energy $\left(E_{b}\right)$ which is defined as

$$
E_{b}=E_{\text {Complex }}-E_{\text {Phos }}-E_{\text {Metal }} \text {, }
$$

where $E_{\text {Complex }}, E_{\text {Phos }}$, and $E_{\text {Metal }}$ are the total energies of metal-doped phosphorene, naked phosphorene, and isolate metal atom, respectively. According to this definition, a more negative value of $E_{b}$ indicates a more energetically favourable (exothermic) binding. As indicated in Table 1, Au atom can form the most stable binding with phosphorene with $E_{b}=-2.31 \mathrm{eV} / \mathrm{Au}$, followed by $\mathrm{Cu}$ and $\mathrm{Li}$. Although the values of $E_{b}$ for $\mathrm{Ag}$ and $\mathrm{Na}$ are relatively smaller, they still reach $-1.24 \mathrm{eV} / \mathrm{Ag}$ and $-1.37 \mathrm{eV} / \mathrm{Na}$, indicating stable adsorption. Generally, the negative values of $E_{b}$ for the five elements under examination indicate that metal binding on phosphorene is exothermic and chemical bonding in nature.

3.2. Electronic Properties of $\mathrm{Cu}, \mathrm{Ag}$, and $\mathrm{Au}$ Doped Phosphorene. Monolayer phosphorene is a semiconductor with 


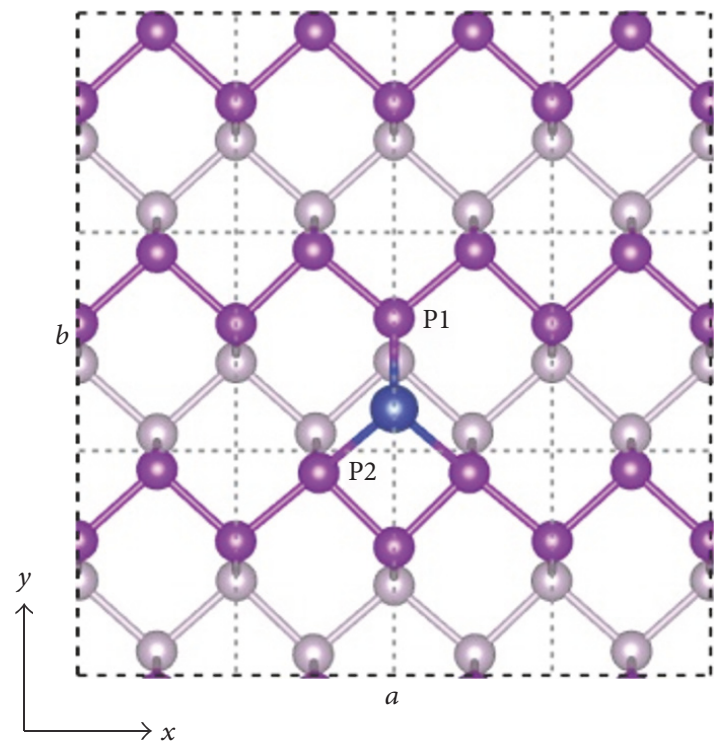

(a)

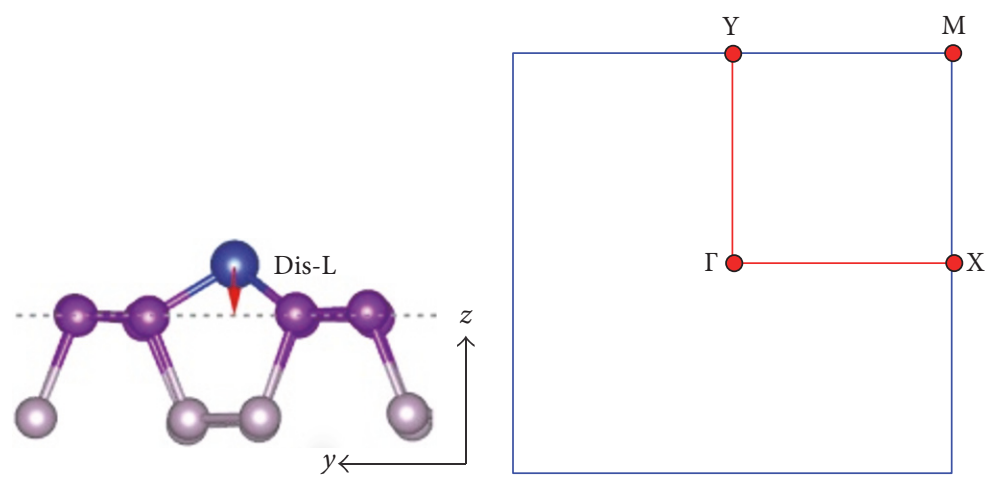

(b)

(c)
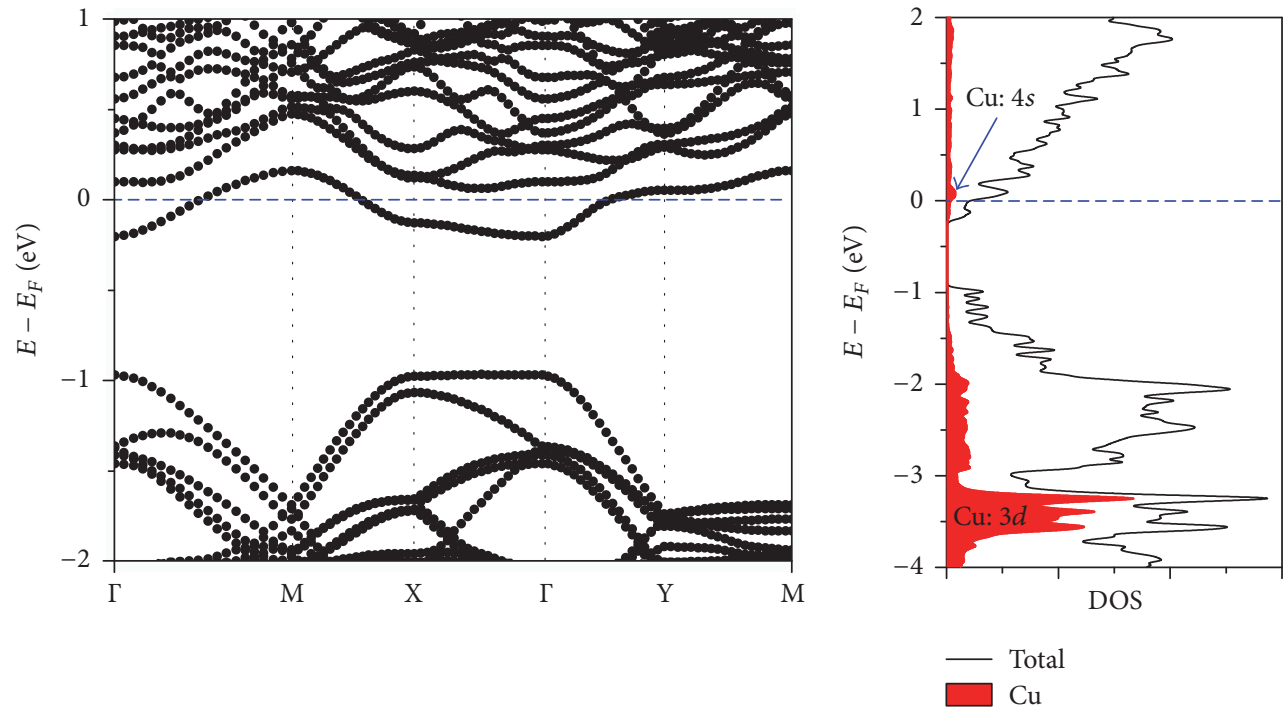

(d)

(e)

Figure 1: ( $\mathrm{a}, \mathrm{b}$ ) Top view and side view of Cu binding on phosphorene; the distance of metal to phosphorene monolayer (Dis-L) is defined as the separation of metal to the upper P atomic layer; (c) the first Brillouin zone and high summary points; (d) electron band structure of phosphorene doped with $\mathrm{Cu}$; (e) density of state of phosphorene doped with $\mathrm{Cu}$ and projection on $\mathrm{Cu}$. In (d) and (e), the blue-colour dash lines indicate the Fermi level.

TABLE 1: Structural parameters and binding energies of metal atoms on phosphorene.

\begin{tabular}{lcccc}
\hline & Distance to P1 $(\AA)$ & Distance to P2 $(\AA)$ & Distance layer $(\AA)$ & Binding energy $(\mathrm{eV} /$ atom $)$ \\
\hline $\mathrm{Cu}$ & 2.22 & 2.34 & 1.01 & -2.13 \\
$\mathrm{Ag}$ & 2.56 & 2.75 & 1.78 & -1.24 \\
$\mathrm{Au}$ & 2.45 & 2.47 & 1.63 & -2.31 \\
$\mathrm{Li}$ & 2.47 & 2.56 & 1.46 & -1.97 \\
$\mathrm{Na}$ & 2.81 & 2.91 & 1.97 & -1.37 \\
\hline
\end{tabular}




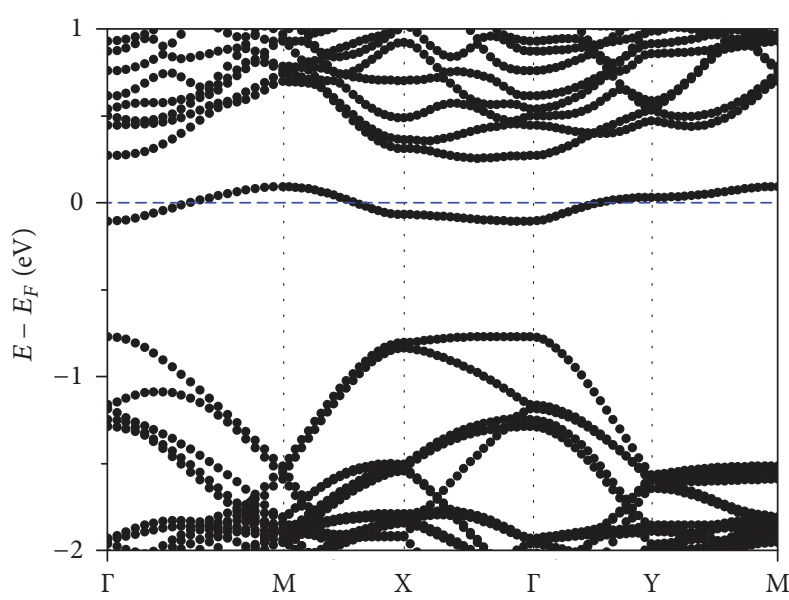

(a)

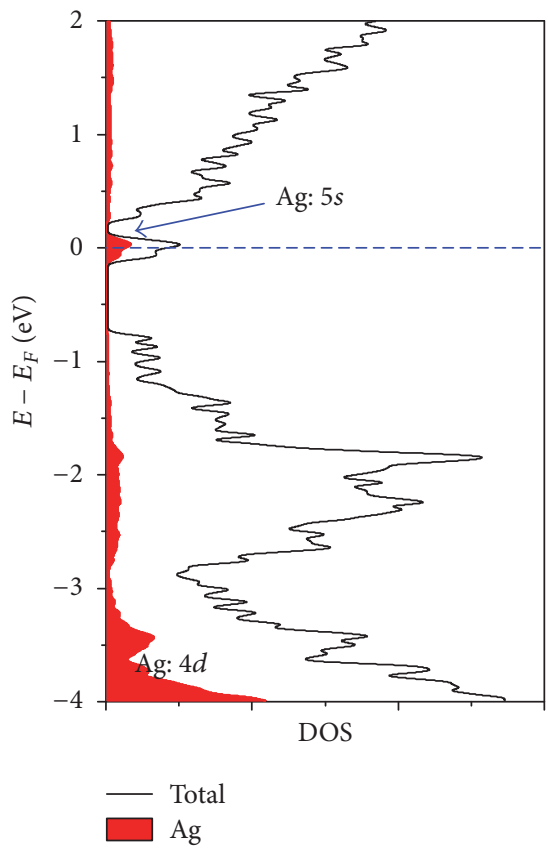

(c)

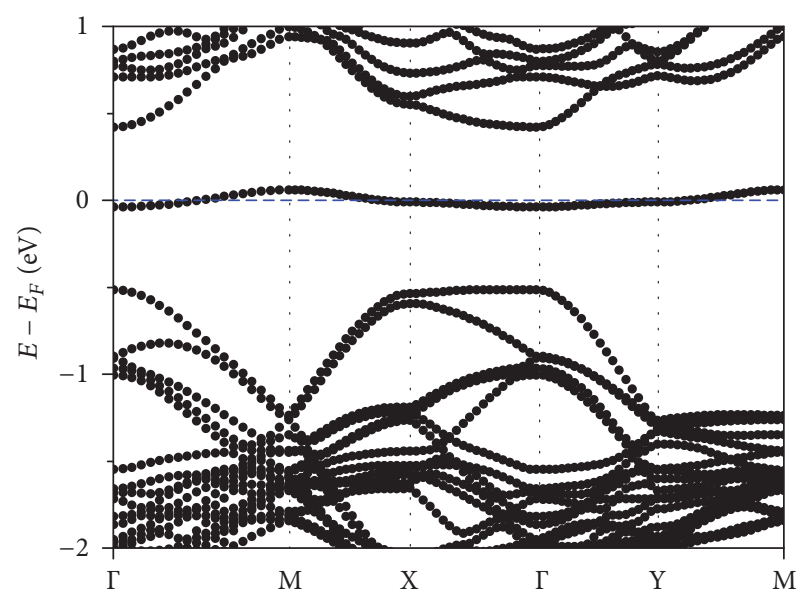

(b)

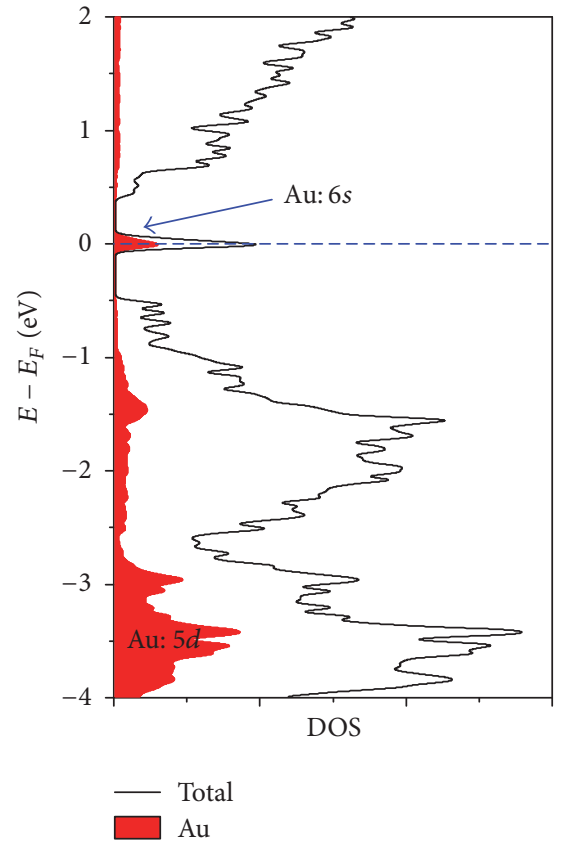

(d)

Figure 2: (a, b) Electronic band structures of $\mathrm{Ag}(\mathrm{a})$ and $\mathrm{Au}(\mathrm{b})$ doped phosphorene; (c, d) density of state of Ag (c) and Au (d) doped phosphorene. The blue-colour dash lines indicate the Fermi level.

a direct bandgap. With $\mathrm{Cu}$ adsorption, significant changes happened to the electronic band structures as shown in Figure 1(d). It is found that $E_{F}$ is upshifted in energy into the bottom of conduction band region, resulting in the degenerate $n$-type semiconducting behaviour. We further checked the electron density of state (DOS) as depicted in Figure 1(e). It is clear that the $3 d$ state of $\mathrm{Cu}$ atom is deep energy level, lying $-3 \mathrm{eV}$ lower than $E_{F}$, while the $4 s$ state of $\mathrm{Cu}$ is located nearby $E_{F}$. However, the states at $E_{F}$ are mainly from phosphorene, indicating strong hybridization between $\mathrm{P}$ and $\mathrm{Cu}$ atoms. Generally, $\mathrm{Cu}$ only contributed by a minor percentage to the states at $E_{F}$; this might point to the fact that the $4 s$ electron of $\mathrm{Cu}$ has been transferred to phosphorene, contributing to the $n$-type semiconducting of the complex (the detailed analyses of electron transfer will be discussed further in Section 3.4).

As can be seen in Figure 2(a), the case of Ag doping on phosphorene can also induce a half-filled band at $E_{F}$. However, compared to $\mathrm{Cu}$ doping, this band is relatively flat in energy and located in the bandgap. These characteristics are even clearer for Au doped phosphorene: the half-filled band becomes even flat in energy and exists in the middle of the bandgap (Figure 2(b)). Spatially, flat bands in the reciprocal space represent localized electron states in the real space. Thus, the flat bands in the $\mathrm{Ag} / \mathrm{Au}$ doped phosphorene should be better categorized as impurity states as the electrons occupying the bands have large effective mass, corresponding to bad conductivity. From DOS analysis (Figures 2(c) and 


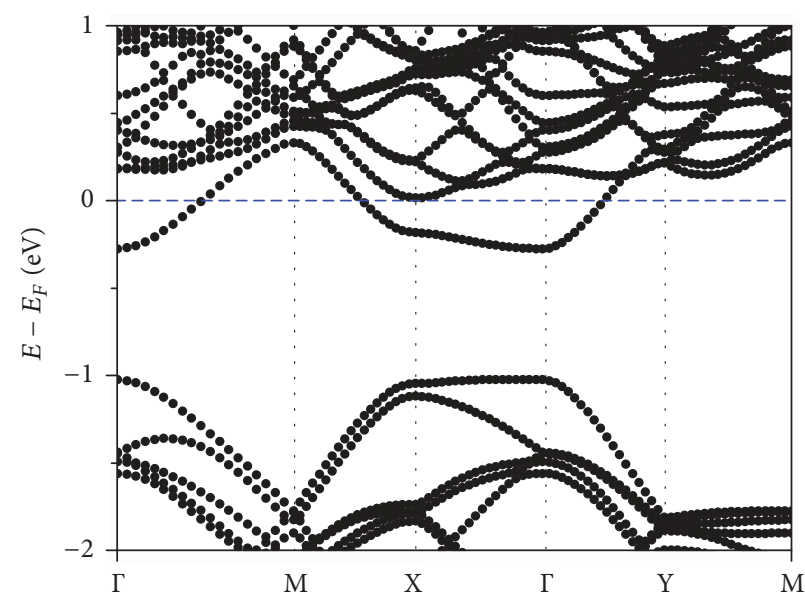

(a)

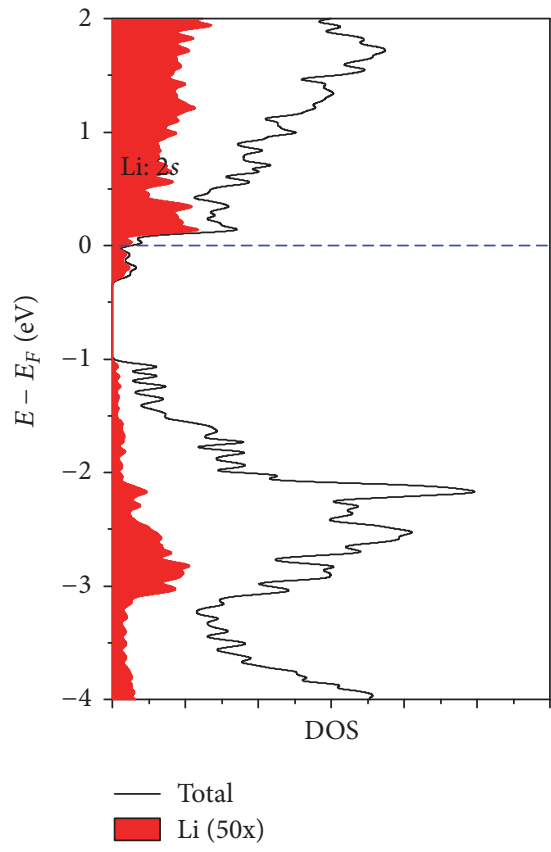

(c)

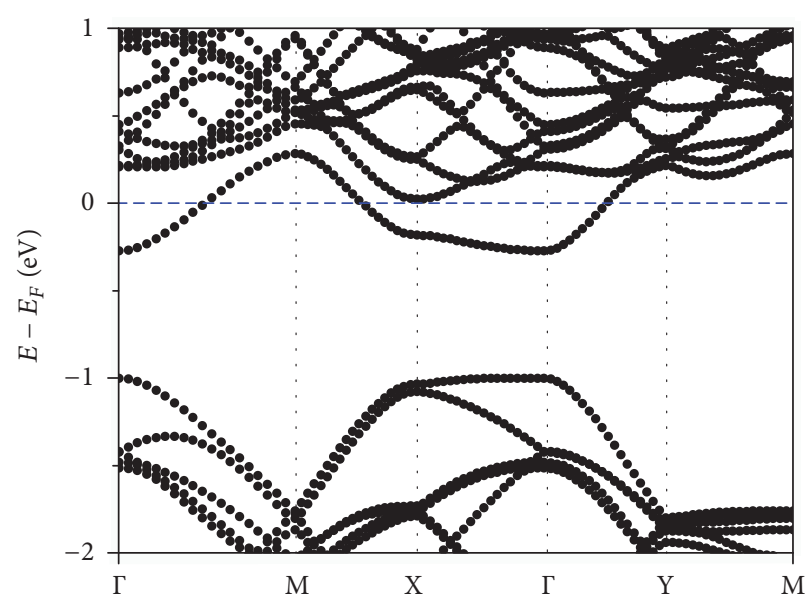

(b)

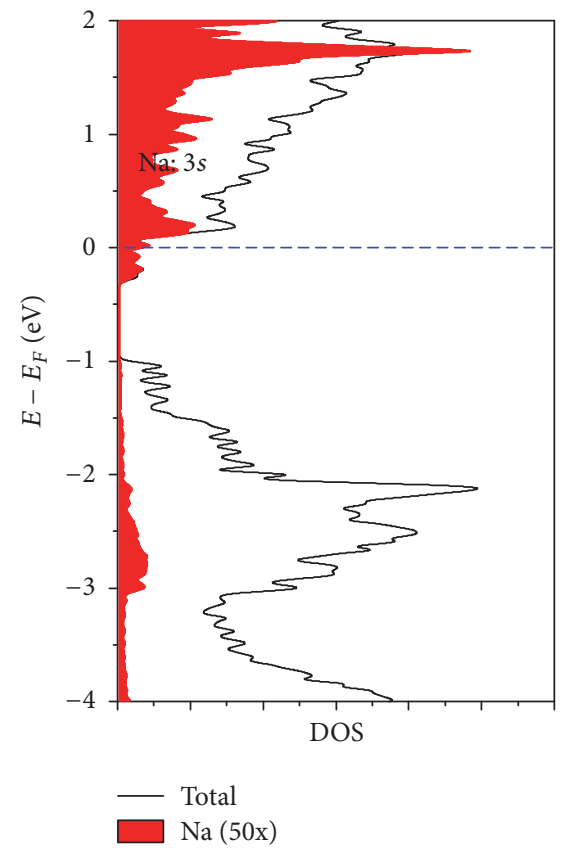

(d)

Figure 3: (a, b) Electronic band structures of $\mathrm{Li}(\mathrm{a})$ and $\mathrm{Na}(\mathrm{b})$ doped phosphorene; (c, d) density of state of Li (c) and Na (d) doped phosphorene; the contributions from $\mathrm{Li}$ and $\mathrm{Na}$ are multiplied by 50 for clear view. The blue-coloured dash lines indicate the Fermi level.

$2(\mathrm{~d}))$, it is clear that the localized $5 s$ of $\mathrm{Ag}$ and $6 s$ of $\mathrm{Au}$ dominate in the states at $E_{F}$. These phenomena are completely different from the $\mathrm{Cu}$ doped phosphorene where $E_{F}$ states spread on phosphorene instead of localizing on $\mathrm{Cu}$ atom. First, this indicates that $\mathrm{Ag}$ and $\mathrm{Au}$ devote fewer electrons than $\mathrm{Cu}$ to the phosphorene. Second, the localized valence electron at the $\mathrm{Ag} / \mathrm{Au}$ atoms flattens the $E_{F}$ band, corresponding to the higher effective mass.

3.3. Verification Study: Li and Na Doped Phosphorene. From the studies of phosphorene doped with $\mathrm{Cu}, \mathrm{Ag}$, and $\mathrm{Au}$, it is clear that the band structures of the composites are highly depending on the species of metal elements, in other words, the hybridization between metal atoms with phosphorene.
The $4 s$ electron of $\mathrm{Cu}$ is easily transferred to phosphorene, resulting in the $n$-type semiconducting. On the contrary, the $6 s$ electron of $\mathrm{Au}$ prefers to locate at $\mathrm{Au}$ atom, resulting in a localized impurity state with massive electron. An interesting hypothesis arises: Is the amount of electron transfer between metal and phosphorene mainly depending on different electronegativity? To answer this question, a verification study is indispensable. We have chosen two alkali elements, Li and $\mathrm{Na}$, considering that they also have one valance electron on the outmost s-orbital, similar to the case of $\mathrm{Cu}, \mathrm{Ag}$, and $\mathrm{Au}$. It is widely accepted that $\mathrm{Li}$ and $\mathrm{Na}$ are highly active to denote their valence electrons to contacting materials [37].

The band structures of phosphorene with $\mathrm{Li}$ and $\mathrm{Na}$ doping are shown in Figures 3(a) and 3(b). It is clear that the 


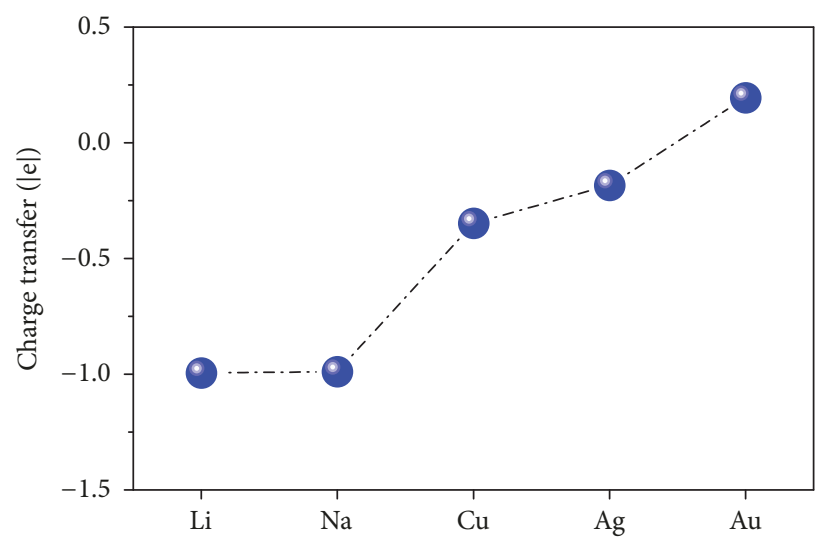

(a)

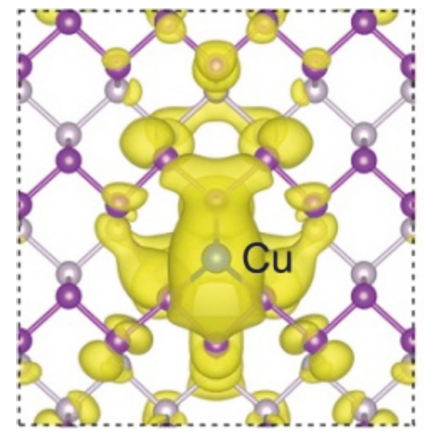

(c)
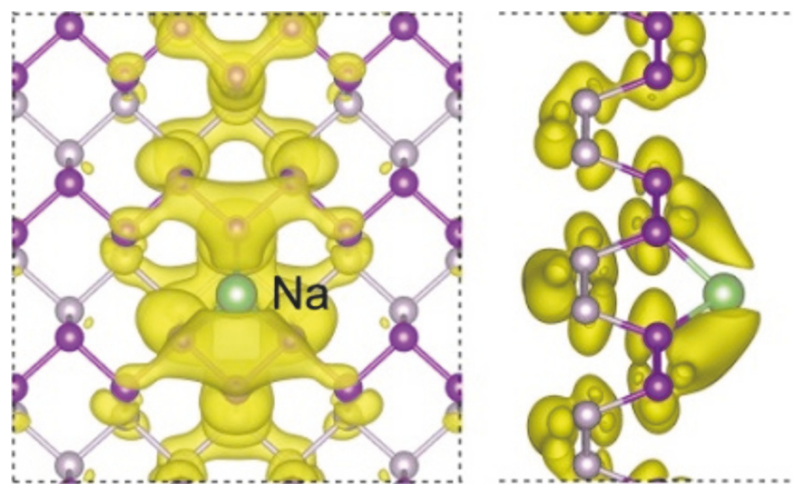

(b)
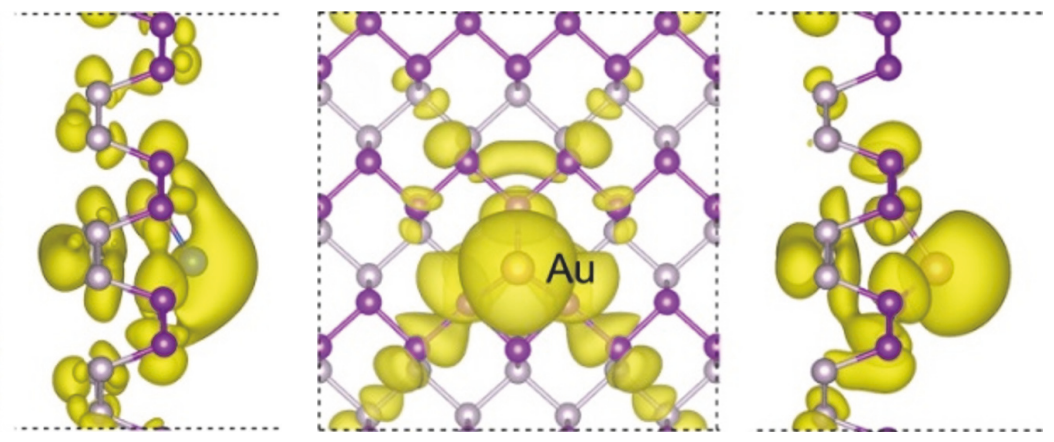

(d)

FIGURE 4: (a) The electron loss of a metal atom when binding to phosphorene (a positive value for Au indicates that electron transfers from phosphorene to $\mathrm{Au})$; (b-d) the spatial distributions of the band at Fermi level of phosphorene doped with $\mathrm{Na}, \mathrm{Cu}$, and $\mathrm{Au}$, respectively (isosurface $=0.0006|\mathrm{e}| / \mathrm{bohr}^{3}$ ).

patterns of the bands at $E_{F}$ are quite similar to the case of $\mathrm{Cu}$ doping. The band at $E_{F}$ is more dispersed in energy than that of $\mathrm{Cu}$ doping, indicating even smaller effective mass of the charge carriers. From DOS analysis in Figures 3(c) and 3(d), it is clear that the states at $E_{F}$ are mostly contributed from phosphorene atoms; the states on $\mathrm{Li} / \mathrm{Na}$ atoms are negligible. This is in line with the case of $\mathrm{Cu}$ doping and supports our hypothesis that the electronegativity of the foreign elements is the key factor.

3.4. Electronegativity and Electron Transfer Analyses. We will now describe the relationship between electronegativity and electron transfer analyses here. In order to quantify the bond polarity between metal atom and phosphorene, we calculated the electron transfer between metal atom and phosphorene with Bader charge analysis toolkit [38]. The values are summarized in Figure 4(a). In detail, $\mathrm{Li}$ and $\mathrm{Na}$ can denote almost all the $s$-electron to phosphorene, while the electron from $\mathrm{Cu}$ to phosphorene is $0.35|\mathrm{e}|$. For $\mathrm{Ag}$, there is only $0.18|\mathrm{e}|$ transferred to phosphorene. For Au with phosphorene, the condition is reversed: $\mathrm{Au}$ atom receives 0.19 |e| from phosphorene, indicating the strongest electron attracting capability. We find that the different amount of electron transfer between metal atom and phosphorene is directly determined by the electronegativity. In detail, the electronegativity order in Pauling units is $\mathrm{Li}(0.93)<\mathrm{Na}(1.0)$
$<\mathrm{Cu}(1.9)<\mathrm{Ag}(1.93)<\mathrm{P}(2.19)<\mathrm{Au}(2.54)$. This order is well consistent with the findings from the above electron transfer analyses, especially that Au can attract electron from phosphorene.

Spatially, the electron distributions corresponding to the states at $E_{F}$ are summarized in Figures 4(b)-4(d). For Na in Figure 4(b), as the $3 s^{1}$ electron of $\mathrm{Na}$ is completely transferred to phosphorene atom, the electron state is absent at Na position but spreads at phosphorene along the armchair direction, constituting the electron conducting channel. Phosphorene doped with $\mathrm{Cu}$ is in a moderate manner (Figure 4(c)): although certain electron state is found at $\mathrm{Cu}$ atoms, there is still considerable electron located at phosphorene. Hence the valence electron still has good mobility and constitutes the $n$-type semiconducting of the complex. On the contrary, as $\mathrm{Au}$ is even more electronegative than $\mathrm{P}$, the bonding electrons are drawn away from $\mathrm{P}$ and localized at the $\mathrm{Au}$ position as illustrated in Figure 4(d), while the electron state at phosphorene is negligible. These results might reveal a catalytic ability of phosphorene with Au coverage because of enhanced electron state at $\mathrm{Au}$. From previous studies, $\mathrm{CeO}_{2}$ substrate was reported to effectively increase the activity of Au clusters for CO oxidation by up to two orders $[39,40]$.

3.5. Cu Coverage Ratio Effect. To ensure the validity of the $n$ type doping by $\mathrm{Cu}$, we have considered two higher coverage 


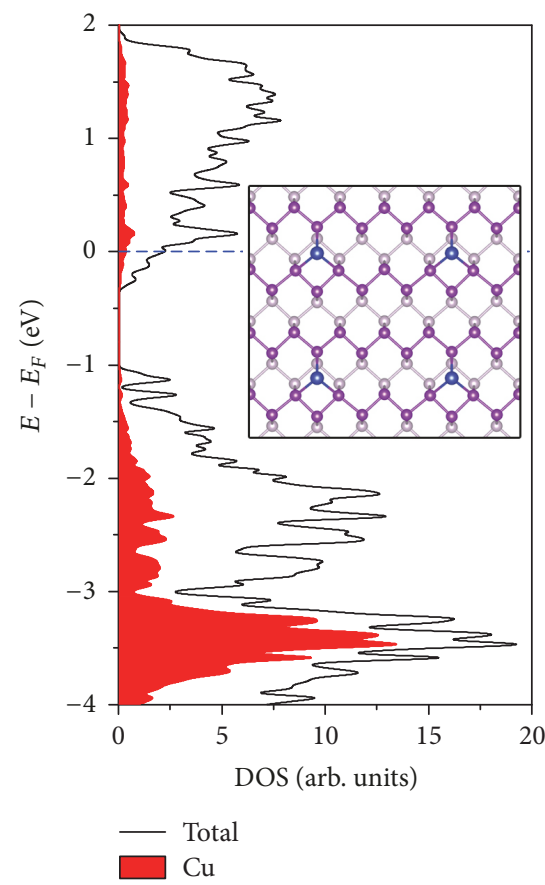

(a)

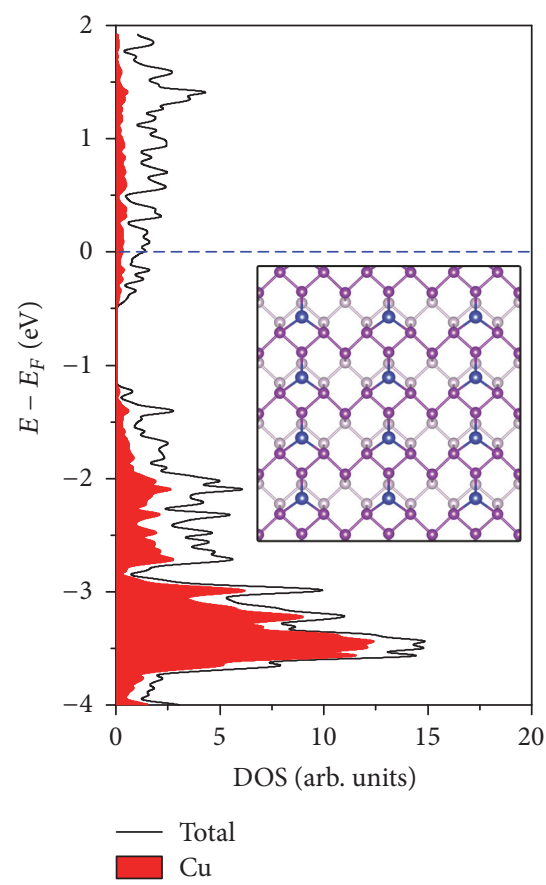

(b)

FiguRE 5: Electron density of states of phosphorene doped with $\mathrm{Cu}$ at coverage ratios of (a) $4.16 \%$ and (b) $12.5 \%$. The blue-coloured dash lines indicate the Fermi level.

ratios of $\mathrm{Cu}$ : one $\mathrm{Cu}$ in a $3 \times 2$ supercell and one $\mathrm{Cu}$ in a 2 $\times 1$ supercell. These correspond to $\mathrm{Cu}$ atomic concentrations of $4.16 \%$ and $12.5 \%$, respectively. The DOS of these two cases are summarized in Figure 5. It is obvious that the value of $E_{F}$ is upshifting with respect to increase of $\mathrm{Cu}$ coverage ratio. This is because more electrons are transferred to phosphorene when $\mathrm{Cu}$ ratio is increased. Thus, the complex will become metallic at high $\mathrm{Cu}$ coverage ratios.

3.6. Work Function Study. From above analysis, the metal atom adsorbents can effectively regulate the band structures of phosphorene resulting in diverse electronic properties. For semiconductors used in electronic devices and circuits, the work function is an important parameter for their real applications, because it dictates the charge transfer direction at interface [41]. Hence we have further examined the work functions of the metal-doped phosphorene systems and pristine phosphorene. Here, the work function is determined as the energy difference between the Fermi level and the vacuum level (the electrostatic potential in the middle of vacuum) $[42,43]$. Following previous work, the Fermi level of pristine phosphorene is set at the mid-gap [42]. As metal atom on phosphorene induces charge transfer between $\mathrm{P}$ and metal atoms, dipole corrections have been taken into consideration in our calculations.

As shown in Figure 6, the pristine phosphorene has a work function of $4.61 \mathrm{eV}$ from our present calculation. This is consistent with previous report of 4.60 [44] and $4.62 \mathrm{eV}$ [45]. Generally, the work functions of the metal-doped phosphorene uniformly decrease. Among the five metal elements, it is found that $\mathrm{Na}$ doping induces the most dramatic decrease of

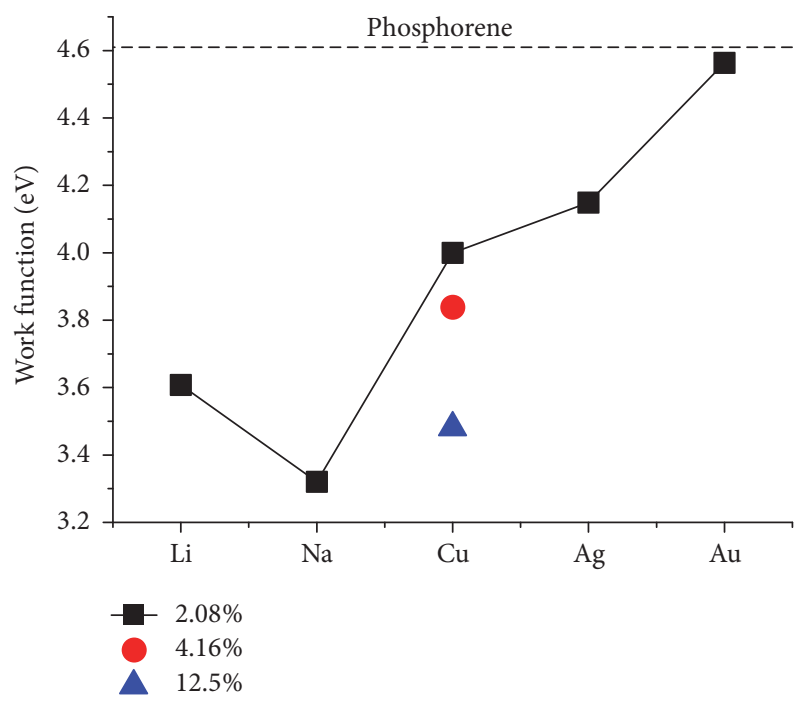

FIGURE 6: Work functions of phosphorene doped with five metal elements. The dash line indicates the work function of pristine phosphorene.

the work function to a value of only $3.32 \mathrm{eV}$. The Li doping also results in significant decrease of work function from $4.61 \mathrm{eV}$ to $3.61 \mathrm{eV}$. From previous analyses, $\mathrm{Li}$ and $\mathrm{Na}$ can effectively transfer electrons to phosphorene; thus the doping site can be treated as an effective dipole on phosphorene surface. The $z$-component (the normal direction of phosphorene layer) of the dipole moment affects the work function. From our calculations, the effective dipole for $\mathrm{Li}$ doping site is 
$0.48|\mathrm{e}| \cdot \AA$. For $\mathrm{Na}$, the dipole is larger, $0.79|\mathrm{e}| \cdot \AA$. For $\mathrm{Cu}$ and $\mathrm{Ag}$, they are also capable of decreasing the work function of phosphorene to a value of 4.00 and $4.15 \mathrm{eV}$, respectively. For the two higher $\mathrm{Cu}$ doping concentrations, the work functions are further decreased to 3.83 and $3.48 \mathrm{eV}$ at $\mathrm{Cu}$ concentrations of $4.16 \%$ and $12.5 \%$, respectively. Thus, it can be concluded that the work function is inversely dependent on doping concentration. Among all the five metal elements, it is interesting to find that Au doping can hardly regulate the work function of phosphorene, which is still $4.56 \mathrm{eV}$, very close to that of pristine phosphorene.

\section{Conclusions}

In general, through density functional theory calculations, we have explored the electronic structure engineering of phosphorene doped with five metal elements: $\mathrm{Cu}, \mathrm{Ag}, \mathrm{Au}, \mathrm{Li}$, and Na. All the five metal atoms can form stable chemical binding with phosphorene atoms. It is interesting to find that $\mathrm{Cu}$ doping can induce the degenerate $n$-type semiconducting for phosphorene among the three group 11 elements. On the contrary, Ag and Au can introduce formation of localized impurity states. Parallel studies of phosphorene doped with $\mathrm{Li}$ and $\mathrm{Na}$ also result in well-behaved $n$-type semiconducting. We attribute the different doping of phosphorene to the compatible electronegativity of doping atoms: $\mathrm{Li}, \mathrm{Na}$, and $\mathrm{Cu}$ have smaller electronegativity than $\mathrm{P}$ and can denote their valence electron to phosphorene. This effectively shifts the Fermi level into the bottom of conduction band region resulting in the degenerate $n$-type semiconducting. On the contrary, Ag and Au have larger electronegativity (Au's electronegativity is even larger than $\mathrm{P}$ ), can hardly transfer electrons to phosphorene, and thus can only introduce localized impurity states. Work function studies indicate that $\mathrm{Cu}$ doping can effectively regulate the work function of phosphorene, which gradually decreases upon increasing $\mathrm{Cu}$ concentration. It is also interesting to find that, among the five metal elements, $\mathrm{Au}$ is almost incapable of changing phosphorene's work function. Summarizing our findings, $\mathrm{Cu}$ becomes the most promising candidate to induce $n$-type semiconducting of phosphorene in the experiment.

\section{Conflicts of Interest}

The authors declare that they have no conflicts of interest.

\section{Acknowledgments}

This work is supported by National Natural Science Foundation of China (21405108 and 11674264), National Key Research and Development Program (2016YFB0201404), Natural Science Foundation of Shandong Province (ZR2015YL017), and Shandong Provincial Outstanding Research Award Fund for Young Scientists of China (BS2014CL046).

\section{References}

[1] K. S. Novoselov, A. K. Geim, S. V. Morozov et al., "Electric field in atomically thin carbon films," Science, vol. 306, no. 5696, pp. 666-669, 2004.
[2] Y. Zhang, Y.-W. Tan, H. L. Stormer, and P. Kim, "Experimental observation of the quantum hall effect and Berry's phase in graphene," Nature, vol. 438, no. 7065, pp. 201-204, 2005.

[3] A. G. I. Grigorieva, "Van der Waals heterostructures," Nature, vol. 499, no. 7459, pp. 419-425, 2013.

[4] M. Du, X. Li, A. Wang, Y. Wu, X. Hao, and M. Zhao, “One-step exfoliation and fluorination of boron nitride nanosheets and a study of their magnetic properties," Angewandte Chemie, vol. 126, no. 14, pp. 3719-3723, 2014.

[5] P. Miro, M. Audiffred, and T. Heine, "An atlas of twodimensional materials," Chemical Society Reviews, vol. 43, no. 18, pp. 6537-6554, 2014.

[6] C.-C. Liu, W. Feng, and Y. Yao, "Quantum spin Hall effect in silicene and two-dimensional germanium," Physical Review Letters, vol. 107, no. 7, Article ID 076802, 2011.

[7] B. Feng, Z. Ding, S. Meng et al., "Evidence of silicene in honeycomb structures of silicon on Ag(111)," Nano Letters, vol. 12, no. 7, pp. 3507-3511, 2012.

[8] F.-F. Zhu, W.-J. Chen, Y. Xu et al., "Epitaxial growth of twodimensional stanene," Nature Materials, vol. 14, no. 10, pp. 10201025, 2015.

[9] K. F. Mak, C. Lee, J. Hone, J. Shan, and T. F. Heinz, "Atomically thin $\mathrm{MoS}_{2}$ : a new direct-gap semiconductor," Physical Review Letters, vol. 105, no. 13, Article ID 136805, 2010.

[10] B. Radisavljevic, A. Radenovic, J. Brivio, V. Giacometti, and A. Kis, "Single-layer $\mathrm{MoS}_{2}$ transistors," Nature Nanotechnology, vol. 6, no. 3, pp. 147-150, 2011.

[11] P. Vogt, P. De Padova, C. Quaresima et al., "Silicene: compelling experimental evidence for graphenelike two-dimensional silicon," Physical Review Letters, vol. 108, no. 15, Article ID 155501, 2012.

[12] C. R. Dean, A. F. Young, I. Meric et al., "Boron nitride substrates for high-quality graphene electronics," Nature Nanotechnology, vol. 5, no. 10, pp. 722-726, 2010.

[13] J. V. Lauritsen, J. Kibsgaard, S. Helveg et al., "Size-dependent structure of $\mathrm{MoS}_{2}$ nanocrystals," Nature Nanotechnology, vol. 2, no. 1, pp. 53-58, 2007.

[14] J. Qiao, X. Kong, Z.-X. Hu, F. Yang, and W. Ji, "High-mobility transport anisotropy and linear dichroism in few-layer black phosphorus," Nature Communications, vol. 5, article no. 4475, 2014.

[15] L. Li, J. Kim, C. Jin et al., "Direct observation of the layerdependent electronic structure in phosphorene," Nature Nanotechnology, vol. 12, no. 1, pp. 21-25, 2017.

[16] L. Li, Y. Yu, G. J. Ye et al., "Black phosphorus field-effect transistors," Nature Nanotechnology, vol. 9, no. 5, pp. 372-377, 2014.

[17] H. Liu, A. T. Neal, Z. Zhu et al., "Phosphorene: an unexplored 2D semiconductor with a high hole mobility," ACS Nano, vol. 8, no. 4, pp. 4033-4041, 2014.

[18] J. Pei, X. Gai, J. Yang et al., "Producing air-stable monolayers of phosphorene and their defect engineering," Nature Communications, vol. 7, Article ID 10450, 2016.

[19] W. Li, G. Zhang, and Y.-W. Zhang, "Electronic properties of edge-hydrogenated phosphorene nanoribbons: a firstprinciples study," The Journal of Physical Chemistry C, vol. 118, no. 38, pp. 22368-22372, 2014.

[20] Y. Cai, G. Zhang, and Y.-W. Zhang, "Electronic properties of phosphorene/graphene and phosphorene/hexagonal boron nitride heterostructures," The Journal of Physical Chemistry C, vol. 119, no. 24, pp. 13929-13936, 2015. 
[21] L. Wang, X. He, J. Li et al., "Nano-structured phosphorus composite as high-capacity anode materials for lithium batteries," Angewandte Chemie International Edition, vol. 51, no. 36, pp. 9034-9037, 2012.

[22] J. Qian, X. Wu, Y. Cao, X. Ai, and H. Yang, "High Capacity and Rate Capability of Amorphous Phosphorus for Sodium Ion Batteries," Angewandte Chemie, vol. 125, no. 17, pp. 4731-4734, 2013.

[23] M. C. Stan, J. V. Zamory, S. Passerini, T. Nilges, and M. Winter, "Puzzling out the origin of the electrochemical activity of black $\mathrm{P}$ as a negative electrode material for lithium-ion batteries," Journal of Materials Chemistry A, vol. 1, no. 17, pp. 5293-5300, 2013.

[24] J. Sun, G. Zheng, H.-W. Lee et al., "Formation of stable phosphorus-carbon bond for enhanced performance in black phosphorus nanoparticle-graphite composite battery anodes," Nano Letters, vol. 14, no. 8, pp. 4573-4580, 2014.

[25] W. Li, Y. Yang, G. Zhang, and Y.-W. Zhang, "Ultrafast and directional diffusion of lithium in phosphorene for highperformance lithium-ion battery," Nano Letters, vol. 15, no. 3, pp. 1691-1697, 2015.

[26] Y. Deng, Z. Luo, N. J. Conrad et al., "Black PhosphorusMonolayer $\mathrm{MoS}_{2}$ van der Waals Heterojunction p-n Diode," ACS Nano, vol. 8, no. 8, pp. 8292-8299, 2014.

[27] S. P. Koenig, R. A. Doganov, L. Seixas et al., "Electron doping of ultrathin black Phosphorus with Cu Adatoms," Nano Letters, vol. 16, no. 4, pp. 2145-2151, 2016.

[28] M. I. Stockman, "Dark-hot resonances," Nature, vol. 467, no. 7315, pp. 541-542, 2010.

[29] M. W. Knight, H. Sobhani, P. Nordlander, and N. J. Halas, "Photodetection with active optical antennas," Science, vol. 332, no. 6030, pp. 702-704, 2011.

[30] Z. Fang, Y. Wang, Z. Liu et al., "Plasmon-induced doping of graphene," ACS Nano, vol. 6, no. 11, pp. 10222-10228, 2012.

[31] Y. Kang, S. Najmaei, Z. Liu et al., "Plasmonic hot electron induced structural phase transition in a $\mathrm{MoS}_{2}$ monolayer," Advanced Materials, vol. 26, no. 37, pp. 6467-6471, 2014.

[32] W. Li, M. Guo, G. Zhang, and Y.-W. Zhang, "Edge-specific $\mathrm{Au} / \mathrm{Ag}$ functionalization-induced conductive paths in armchair $\mathrm{MoS}_{2}$ nanoribbons," Chemistry of Materials, vol. 26, no. 19, pp. 5625-5631, 2014.

[33] G. Kresse and J. Furthmuller, "Efficient iterative schemes for ab initio total-energy calculations using a plane-wave basis set," Physical Review B: Condensed Matter and Materials Physics, vol. 54, no. 16, pp. 11169-11186, 1996.

[34] G. Kresse and J. Furthmüller, "Efficiency of ab-initio total energy calculations for metals and semiconductors using a plane-wave basis set," Computational Materials Science, vol. 6, no. 1, pp. 15-50, 1996.

[35] P. E. Blöchl, "Projector augmented-wave method," Physical Review B: Condensed Matter and Materials Physics, vol. 50, no. 24, pp. 17953-17979, 1994.

[36] J. P. Perdew, J. A. Chevary, S. H. Vosko et al., "Atoms, molecules, solids, and surfaces: Applications of the generalized gradient approximation for exchange and correlation," Physical Review B: Condensed Matter and Materials Physics, vol. 46, no. 11, pp. 6671-6687, 1992.

[37] P. E. Mason, F. Uhlig, V. Vaněk, T. Buttersack, S. Bauerecker, and P. Jungwirth, "Coulomb explosion during the early stages of the reaction of alkali metals with water," Nature Chemistry, vol. 7, no. 3, pp. 250-254, 2015.
[38] W. Tang, E. Sanville, and G. Henkelman, "A grid-based Bader analysis algorithm without lattice bias," Journal of Physics: Condensed Matter, vol. 21, no. 8, Article ID 084204, 2009.

[39] S. Carrettin, P. Concepción, A. Corma, J. M. López Nieto, and V. F. Puntes, "Nanocrystalline $\mathrm{CeO}_{2}$ increases the activity of Au for CO oxidation by two orders of magnitude," Angewandte Chemie International Edition, vol. 43, no. 19, pp. 2538-2540, 2004.

[40] H. Y. Kim, H. M. Lee, and G. Henkelman, "CO oxidation mechanism on $\mathrm{CeO}_{2}$-supported Au nanoparticles," Journal of the American Chemical Society, vol. 134, no. 3, pp. 1560-1570, 2012.

[41] G. Fiori, F. Bonaccorso, G. Iannaccone et al., "Electronics based on two-dimensional materials," Nature Nanotechnology, vol. 9, no. 10, pp. 768-779, 2014.

[42] B. Shan and K. Cho, "First principles study of work functions of single wall carbon nanotubes," Physical Review Letters, vol. 94, no. 23, Article ID 236602, 2005.

[43] B. Shan and K. Cho, "First-principles study of work functions of double-wall carbon nanotubes," Physical Review B: Condensed Matter and Materials Physics, vol. 73, no. 8, Article ID 081401, 2006.

[44] V. V. Kulish, O. I. Malyi, C. Persson, and P. Wu, "Adsorption of metal adatoms on single-layer phosphorene," Physical Chemistry Chemical Physics, vol. 17, no. 2, pp. 992-1000, 2015.

[45] J. E. Padilha, A. Fazzio, and A. J. R. Da Silva, "Van der Waals heterostructure of phosphorene and graphene: Tuning the schottky barrier and doping by electrostatic gating," Physical Review Letters, vol. 114, no. 6, Article ID 066803, 2015. 


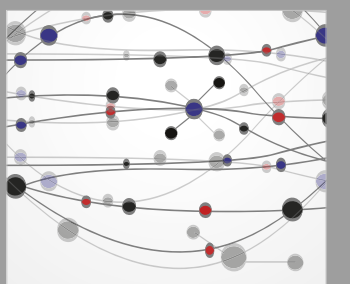

The Scientific World Journal
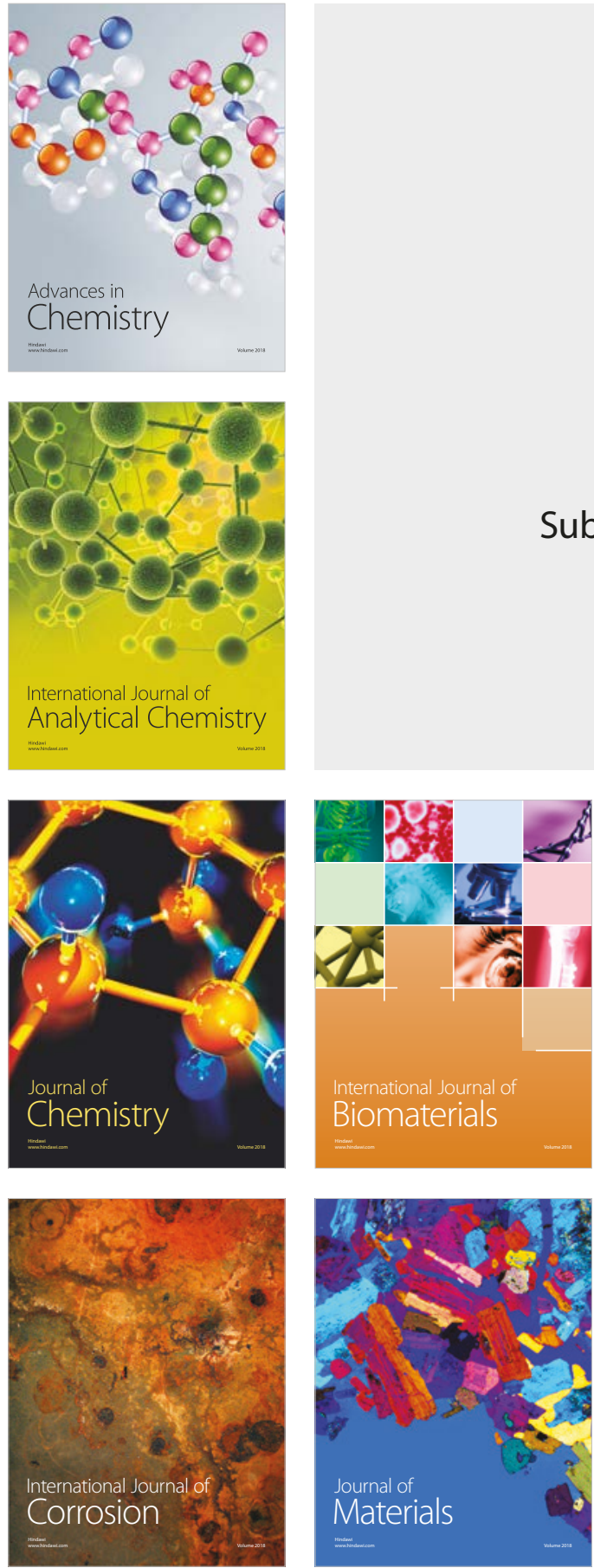

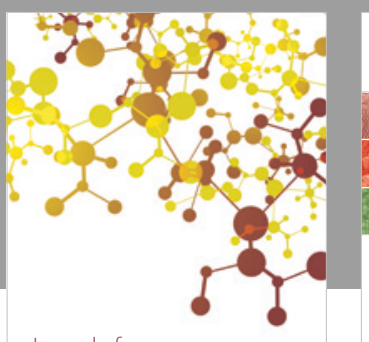

Journal of

Applied Chemistry
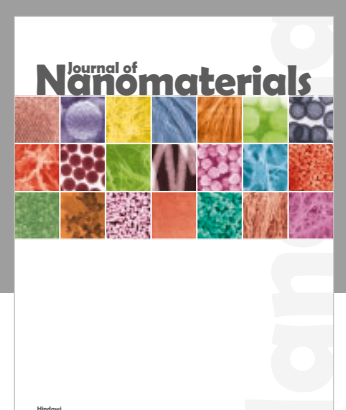

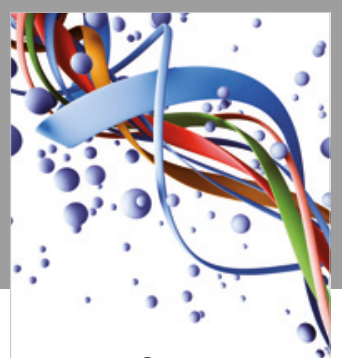

Scientifica

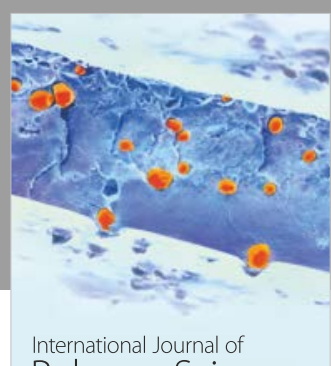

Polymer Science

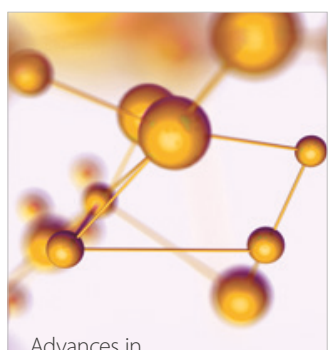

Physical Chemistry
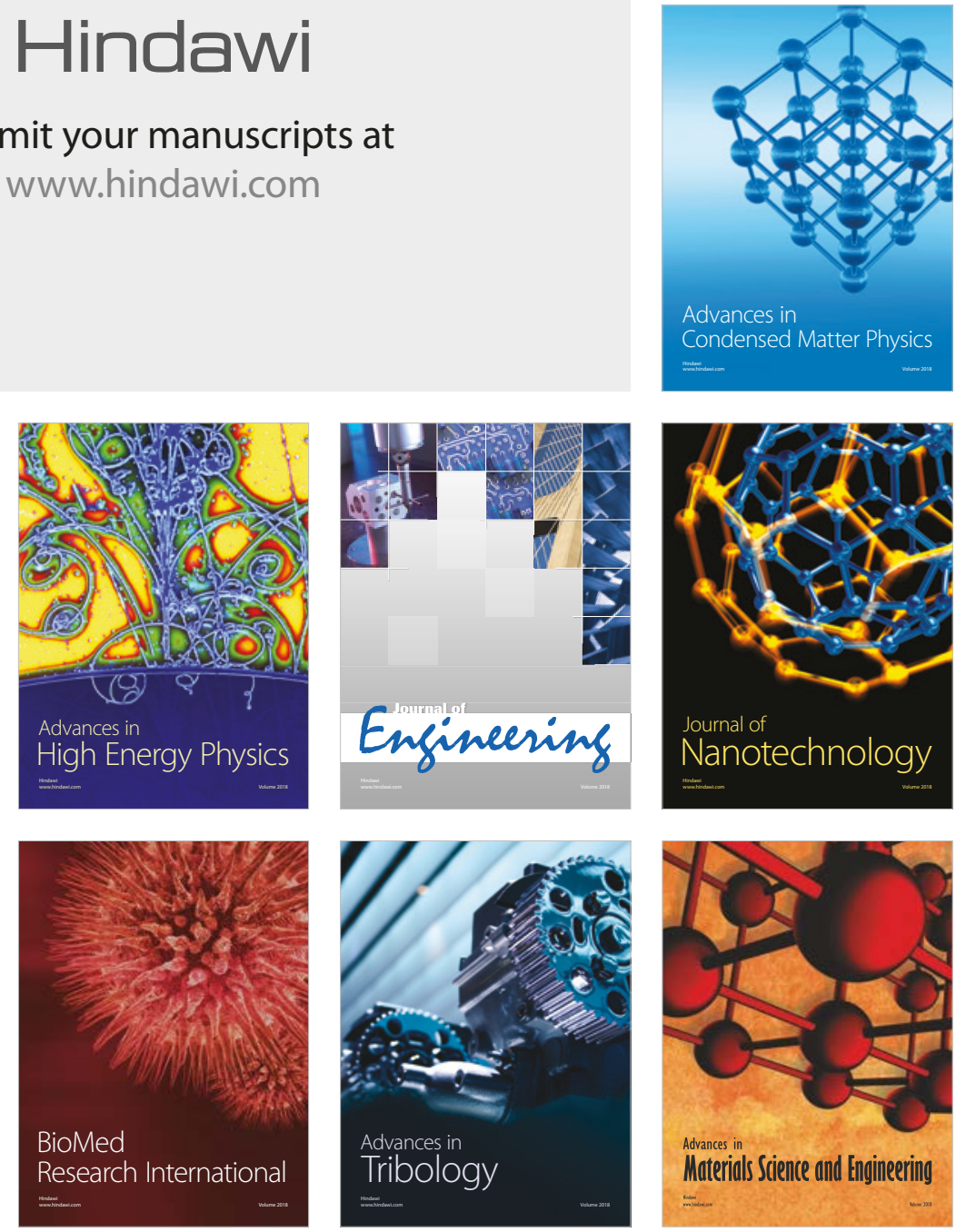